$$
+24 \frac{\varepsilon}{10},+23 \frac{\varepsilon}{10},+\cdots,+16 \frac{\varepsilon}{10},+15 \frac{\varepsilon}{10}
$$

Die entsprechende Reihe bekommen wir für $-2 \varepsilon$. Die o kann nun in der That auch positiv oder negativ sein, jedesmal repräsentirt sie aber nur die Hälfte der Fălle, wie die andern Zahlen, nämlich z. B. +o $\varepsilon$ :

$$
+4 \frac{\varepsilon}{10},+3 \frac{\varepsilon}{10},+2 \frac{\varepsilon}{10},+1 \frac{\varepsilon}{10},+0 \frac{\varepsilon}{10} .
$$

Demnach ist es wohl berechtigt, die o nur einmal zu zählen.

Es geht dies ebenso deutlich aus einer geometrischen Darstellung hervor, die auch für absolute Fehler Gultigkeit hat. Um uns von der Möglichkeit des Ausgehens nach beiden Seiten von o aus bei der gewöhnlichen Darstellung der Zahlenreihe, wodurch wieder die Meinung erweckt werden könnte, dass o doppelt zu zählen sei, zu befreien, wăhlen wir folgende. Denken wir uns die reelle unendliche Ebene

Berlin 1887 Sept. $r$. von einer unendlichen Schaar paralleler äquidistanter Geraden erfüllt und ziehen wir durch diese Schaar eine unendliche Gerade. Die Schnittpunkte dieser mit den Parallelen stellen uns dann die Zahlenreihe $-\infty, \ldots-1,0,+1, \ldots,+\infty$ dar, wenn wir eine im Endlichen liegende Parallele als die bezeichnen, auf der der Schnittpunkt o liegen soll, während alle Schnittpunkte auf der einen Seite der Parallelen die positiven, auf der anderen die negativen Zahlen vorstellen. Es ist klar, dass o nur einen Schnittpunkt hat, die andern Zahlen dagegen zwei. Auch ist wohl kein Grund ersichtlich, warum der Schnittpunkt o doppelt zu zählen wäre. Sehen wir die Fehler aber wieder als nicht absolute an, so können wir bei der gewöhnlichen Darstellung der Zahlenreihe in der Länge der Zahlenbildstücke oder in der Anzahl der etwa in Zehntelintervallen auf eine durch den o-Punkt gehende andere Gerade gefällten Lothe die obige Ueberlegung illustriren.

\title{
Schreiben von Herrn Dr. O. Backlund, Mitglied der Akademie in St. Petersburg
} betr. den neugestifteten Schubert'schen Preis.

Fräulein Schubert hat der Kaiserl. Akademie der Wissenschaften ein Kapital geschenkt, um einen Preis, dem General Schubert zu Ehren, für hervorragende Arbeiten auf dem Gebiete der Astronomie und Mathematik zu stiften. Der Preis, der aus einer Geldsumme von 1000 Rubeln besteht, wird alle zwei Jahre, am 12/24. Febr., dem Geburtstage des Generals Schubert, vertheilt, und zwar so, dass das eine Mal der Preis für Abhandlungen in der Astronomie oder in der Mathematik vergeben wird, und das andere Mal als Belohnung für gelehrte Arbeiten, die zur Lösung wichtiger Aufyaben in der theoretischen Astronomie nach Anweisung der Akademie ausgefürt sind.

$\mathrm{Da}$ in Bezug auf die Abhandlungen in der theoretischen Astronomie dieser Preis international ist, so wäre es der Akademie erwünscht, wenn Sie denselben in den A. N. erwähnen wollten.

Ich schicke Ihnen hiermit ein Exemplar der Statuten und theile einen Auszug in Uebersetzung mit.

\$ 6. Zur Betheiligung am Concurs werden Abhandlungen angenommen, die von dem Verfasser selbst oder von einem Mitgliede der Commission, die die Akademịe zur Beurtheilung der Abhandlung ernennt, präsentirt werden.

\$ 7. In der theoretischen Astronomie können Ab. handlungen, die in russischer, lateinischer, französischer, englischer oder deutscher Sprache gedruckt sind, concurriren, wenn sie nicht früher als 5 Jahre vor dem Concurs erschienen sind.

$\$$ 9. ... Die erste Preisvertheilung findet aın $12 / 24$. Febr. 1889 statt. ...

$\$$ I 2. Die Abhandlungen, die concurriren sollen, dürfen nicht später als den $15 / 27$. August desjenigen Jahres eingeliefert werden, welches dem Concursjahre vorangeht.

$\$$ 13. Nach dem Ablauf dieses 'Termins ernennt die physisch-mathematische Abtheilung der Akademie aus ihren wirklichen Mitgliedern eine Commission, die zuerst zu beurtheilen und mit Stimmenmehrheit zu entscheiden hat, ob mit dem Sciubert'schen Preise irgend eine theoretisch-astronomische Abhandlung liberhaupt gekrönt werden kann. Für den Fall, dass diese Frage im negativen Sinne entschiedeu wird, geht die Commission zur Beurtheilung und Entscheidung über die Preisvertheilung für mathematische Abhandlungen über. St. Petersburg 1887 Sept. 27.

\section{Ueber die Eigenbewegung von Lal. 28607.}

Dr. W. Luther in Hamburg macht mich darauf aufmerksam, dass er die Eigenbewegung von Lal. 28607 (vergl. Kam A. N. 2806) bereits früher in seiner Inaugural-Dissertation: ,Ueber die Bahn des Planeten Amalthea Leipzig 1883 eingehend untersucht habe. Der daselbst S. 26 für die Eigenbewegung angegebene Werth -0.078 in $A \Gamma$.. und -0.28 in $\mathrm{Decl}$. ist mit dem von Kam gefundenen nahezu identisch.

Kr.

\section{Berichtigung zu von Oppolzer's Lehrbuch zur Bahnbestimmung.}

In von Oppolzer's , Lehrbuch zur Bahnbestimmung I, 2. Auflage, befindet sich Seite 1 2, zu Anfang des Abschnittes -Transformation der ekliptischen Coordinaten in aequatoreale und umgekehrte, wahrscheinlich durch das Ausfallen-einer halben Zeile veranlasst, ein Versehen, welches bei aufmerksamem Lesen allerdings unschwer zu entdecken ist, auf welches aufmerksam zu machen aber trotzdem nicht ganz überflüssig sein dürfte. - Es ist nämlich an der genannten Stelle zu lesen : Bezeichnet man die rechtwinkligen A e qua to r e a l coordinaten anstatt wie bisher Ekliptikal coordinaten mit $x^{\prime} y^{\prime} z^{\prime}$. 